\title{
Potential role of hypothalamic microRNAs in regulation of FOS and FTO expression in response to hypoglycemia
}

\author{
Bashair M. Mussa ${ }^{1}$ Jalal Taneera ${ }^{2} \cdot$ Abdul Khader Mohammed $^{3} \cdot$ Ankita Srivastava $^{3}$ - Debasmita Mukhopadhyay ${ }^{3}$. \\ Nabil Sulaiman ${ }^{4}$
}

Received: 29 April 2019 / Accepted: 6 October 2019 / Published online: 14 November 2019

(C) The Author(s) 2019

\begin{abstract}
Hypoglycemia-associated autonomic failure (HAAF) is a serious complication of diabetes which is associated with the absence of physiological homeostatic counter-regulatory mechanisms that are controlled by the hypothalamus and sympathetic nervous system. Identification of biomarkers for early detection of HAAF requires an advanced understanding of molecular signature of hypoglycemia which is yet to be identified. The outcomes of the present study have shown that the viability and the apoptotic rate of the hypothalamic neurons (mHypoE-N39) were decreased significantly due to hypoglycemia in a dose-dependent fashion $(p<0.05)$. Although there are more than 1000 miRNAs differentially expressed in hypothalamus, only twelve miRNAs (miR-7a, miR-7b, miR-9, miR-29b, miR-29c, miR-30a, miR-30b, miR-30c, miR-101b-3p, miR-181a-5p, miR-378-3p and miR-873-5p) were correlated to two main hypothalamic regulatory proteins, FOS and FTO. Expression of these proteins was very sensitive to hypoglycemia. We demonstrated that hypoglycemia modulates the expression of hypothalamic miRNAs that are related to FOS and FTO.
\end{abstract}

Keywords FOS $\cdot$ FTO $\cdot$ microRNAs $\cdot$ Diabetes mellitus $\cdot$ Hypoglycemia-associated autonomic failure

Bashair M. Mussa

bmussa@sharjah.ac.ae

Jalal Taneera

jtaneera@sharjah.ac.ae

Abdul Khader Mohammed

amohammed@sharjah.ac.ae

Ankita Srivastava

ankita2112@gmail.com

Debasmita Mukhopadhyay

debasmitag2009@gmail.com

Nabil Sulaiman

nsulaiman@sharjah.ac.ae

1 Basic Medical Science Department, College of Medicine, University of Sharjah, P.O. Box: 27272, Sharjah,

United Arab Emirates

2 Basic Medical Science Department, College of Medicine, Sharjah Institute for Medical Research, University of Sharjah, P.O. Box: 27272, Sharjah, United Arab Emirates

3 Sharjah Institute for Medical Research, University of Sharjah, P.O. Box: 27272, Sharjah, United Arab Emirates

4 Family Medicine and Behavioral Science, College of Medicine, University of Sharjah, P.O. Box: 27272, Sharjah, United Arab Emirates

\begin{abstract}
Abbreviations
CRR Counter-regulatory response

DM Diabetes mellitus

FTO Fat mass and obesity-associated gene/protein

HAAF Hypoglycemia-associated autonomic failure

ENHs Embryonic hypothalamic neurons
\end{abstract}

\section{Introduction}

Hypothalamus is composed of multiple clusters of neurons which express different hormones, neurotransmitters and proteins. The latter are involved in regulation of a wide range of homeostatic processes, including cognitive functions, glucose sensing and metabolism $[1,2]$.

Interestingly, the functions of the hypothalamus evolve through lifetime to adapt to several external factors including environment, stress and lifestyle [3]. It has been found that aging, exposure to high-fat diet and stressful events influence the hypothalamic functions, dramatically $[4,5]$. In addition, more studies have demonstrated that the threshold of the hypothalamic response is changeable, for instance, the response of leptin threshold to weight change [6]. 
The metabolic processes in the brain are mainly dependent on the peripheral glucose and any reduction in glucose beyond the physiological levels leads to activation of robust counter-regulatory response (CRR) [7]. The latter involves a group of neurons within the hypothalamus and the rostral ventro-lateral medulla which activate the sympathetic system that, in turn, stimulates adreno-medullary response leading to hypoglycemia awareness $[8,9]$. Reduction or absence of CRR leads to hypoglycemia-associated autonomic failure (HAAF) [10]. Given that in diabetes mellitus (DM) the responses of the pancreatic islets to hypoglycemia are disabled, the body mainly depends on autonomic-adreno-medullary regulatory system to correct the hypoglycemia [11].

HAAF is mainly caused by the use of anti-diabetic medications, in particular, insulin-based treatments, with prevalence of $25 \%$ in patients with type 1 DM [12]. The consequences of HAAF range from confusion to convulsions, coma and even death and it also represents a major limitation to achieve an optimal control of DM and this influences the quality of life of patients with DM, adversely [13].

MicroRNAs (miRNAs), non-coding ribonucleic acid (RNA) molecules, negatively regulate the expression of various proteins within the hypothalamus [14]. MiRNAs mediate post-transcriptional silencing of target genes via binding to the $3^{\prime}$-untranslated regions (3'-UTR) of target mRNAs which eventually leads to inhibition of protein synthesis [14]. Hypothalamic miRNAs are involved in control of food intake and energy balance and their dysregulation in early life is associated with insulin resistance in adulthood [15]. It is believed that miRNAs are involved in regulation of metabolic profile via direct interaction with the regulatory hypothalamic proteins [16].

Previous reports have demonstrated changes in the expression of the hypothalamic miRNA after chronic hyperosmolar stimulation which, in turn, influenced the regulation of FOS [17]. In addition, FOS expression has been shown to decrease within the medial and dorsal hypothalamic areas in response to hypoglycemia [18-22]. Given that these hypothalamic areas are involved in the CRR, it was hypothesized that the reduction in FOS expression is an indicator of HAAF [23]. On the other hand, changes in FOS expression in diabetic neuropathy and other stressful events including hypoxia-ischemia suggest a neuro-protective role for FOS expression [24, 25]. Moreover, it has been reported that FOS is involved in regulation of food intake and gastric motility [26]. Therefore, it seems that the involvement of FOS in regulation of the neuronal signal is more complex than previously believed.

Fat mass and obesity-associated protein (FTO) is another protein that is highly expressed in the hypothalamic region with critical roles for control of energy balance in a nutritionally dependent manner [27]. Clinical studies have demonstrated a strong association between a single-nucleotide polymorphism in the FTO gene and obesity in diverse ethnic populations [28, 29]. In addition to its association with body mass index, variations in the FTO gene have been linked with glucose metabolism and type 2 DM $[28,30]$. FTO mRNA is expressed in several tissue types, with most abundant expression in the hypothalamic nuclei, and is involved in regulation of several feeding processes [27, 28].

Taking into account the previous findings, it is crucial to investigate the molecule signature of hypoglycemia and the expression of hypothalamic miRNAs, FOS and FTO simultaneously to propose novel biomarkers for HAAF.

\section{Materials and methods}

\section{Cells and culture conditions}

Embryonic mouse hypothalamus cells (EHNs, mHypoEN39) were obtained from Cedarlane (Burlington, Ontario, Canada) and cultured in Dulbecco's Modified Eagle's Medium (DMEM; Sigma-Aldrich) containing $4500 \mathrm{mg} / \mathrm{L}$ glucose supplemented with 10\% Fetal Bovine Serum (FBS; Sigma-Aldrich), $1 \%$ penicillin/streptomycin (100 units/mL; Sigma-Aldrich) and maintained at $37{ }^{\circ} \mathrm{C}$ with humidified atmosphere of $5 \% \mathrm{CO}_{2}$ and $95 \%$ air. The cells were cultured in $75 \mathrm{~cm}^{2}$ cell culture flask (Sigma-Aldrich). Four different conditions of low glucose were established by seeding the cells in DMEM containing $2000 \mathrm{mg} / \mathrm{L}, 900 \mathrm{mg} / \mathrm{L}, 500 \mathrm{mg} / \mathrm{L}$ and $200 \mathrm{mg} / \mathrm{L}$ of glucose. The low glucose conditions (2000 mg/L glucose) constituted of $44.4 \mathrm{~mL}$ of DMEM containing $4500 \mathrm{mg} / \mathrm{L}$ glucose, and $55.6 \mathrm{~mL}$ of DMEM without glucose. Similarly, low glucose conditions of $900 \mathrm{mg} / \mathrm{L}$, $500 \mathrm{mg} / \mathrm{L}$ and $200 \mathrm{mg} / \mathrm{L}$ were obtained by mixing $20 \mathrm{~mL}$, $11.2 \mathrm{~mL}$ and $4.44 \mathrm{~mL}$ of DMEM containing $4500 \mathrm{mg} / \mathrm{L}$ glucose, and $80 \mathrm{~mL}, 88.88 \mathrm{~mL}$ and $96.4 \mathrm{~mL}$ of DMEM without glucose, respectively. Cells were maintained under these regimens for $72 \mathrm{~h}$; medium was changed every $48 \mathrm{~h}$.

\section{Cell viability}

Cell viability was measured using the 3-[4,5-dimethylthiazol-2-yl]-2,5diphenyltetrazolium bromide (MTT; SigmaAldrich) colorimetric assay. Briefly, 5000 cells were seeded in 96-well plates with $150 \mu \mathrm{L}$ culture medium containing different glucose concentrations $(2000,900,500,200 \mathrm{mg} / \mathrm{L}$ of glucose) and maintained for 24, 48 and $72 \mathrm{~h}$. The media were replaced with $100 \mu \mathrm{L}$ of phosphate-buffered saline (PBS; Sigma-Aldrich) and $20 \mu \mathrm{L}$ of MTT solution $(5 \mathrm{mg} /$ $\mathrm{mL}$ ) and incubated at $37^{\circ} \mathrm{C}$ for $180 \mathrm{~min}$. MTT formzan crystals were dissolved in $100 \mu \mathrm{L}$ of dimethyl sulfoxide (DMSO; Sigma-Aldrich) and absorbance was recorded at $570 \mathrm{~nm}$ on a microplate reader. The percentage of cell viability was calculated from the average $570 \mathrm{~nm}$ absorbance value as 
per the following equation: \% cell viability $=(\mathrm{OD} 570 \mathrm{~nm}$ of sample/OD $570 \mathrm{~nm}$ of control) $\times 100$.

\section{Apoptosis analysis}

For apoptosis analysis, $1 \times 10^{6}$ cells were seeded in a 6-well plate and maintained in different glucose concentrations for $72 \mathrm{~h}$. Apoptosis assay was performed using annexin V-FITC apoptosis staining/detection kit (Abcam). Cells were harvested and washed twice with PBS. Around $0.5 \times 10^{5}$ cells were resuspended in $500 \mu \mathrm{L}$ of $1 \times$ binding buffer followed by adding $3 \mu \mathrm{L}$ of Annexin V-FITC and $3 \mu \mathrm{L}$ propidium iodide and incubated in dark for $10 \mathrm{~min}$ at room temperature. Cells were analyzed by BD Accuri flow cytometer (Becton Dickinson) applying FITC channel (515 nm) and PE detector $(585 \mathrm{~nm})$ and a minimum of 15,000 events were collected for the analysis. Compensation was performed using the single color controls with Annexin FITC and PI.

\section{Total RNA, miRNA extraction and cDNA synthesis}

In a 6 -well plate, $1.5 \times 10^{5}$ cells were seeded and maintained in different glucose concentrations for $72 \mathrm{~h}$. The total RNA was extracted using Pure Link RNA Mini Kit (Invitrogen) following the manufacturer's instruction. MiRNA was extracted using miRNA purification Kit (NorgenBiotek) in accordance with manufacturer's protocol. RNA quantification was performed using nanodrop spectrophotometer and purity was determined by 260/280 ratio. The reverse-transcription for total RNA (approximately $1 \mu \mathrm{g}$ ) was performed in final reaction volume of $20 \mu \mathrm{L}$ containing mixture of 200 units of M-MLV Reverse Transcriptase (Sigma-Aldrich), $10 \mathrm{mM}$ dNTP mix, $5 \mu \mathrm{M}$ random primers and reaction buffer. The reaction mix was incubated at $37^{\circ} \mathrm{C}$ for $50 \mathrm{~min}$ followed by $85^{\circ} \mathrm{C}$ for $10 \mathrm{~min}$. Reverse transcription for miRNA ( $\sim 500 \mathrm{ng})$ was performed with miScript II RT kit (Qiagen) in a final reaction volume of $20 \mu \mathrm{L}$ that contained $4 \mu \mathrm{L}$ of Hispec buffer $(5 \times), 2 \mu \mathrm{L}$ of nucleic mix $(10 \times)$ and $2 \mu \mathrm{L}$ miScprit reverse transcriptase. The reaction mix was then incubated at $37{ }^{\circ} \mathrm{C}$ for $60 \mathrm{~min}$ followed by $90{ }^{\circ} \mathrm{C}$ for $5 \mathrm{~min}$.

\section{Gene expression analysis}

All the qPCR experiments for FOS and FTO gene expression were performed in 48-well plates using StepOne Real-Time PCR system (Applied Biosystems) and a predesigned TaqMan gene expression assays for FOS (assay ID: Mm00487425_m1) and FTO (assay ID: Mm00488755_ m1) gene (Applied Biosystems). The expression of the FOS and FTO mRNA was normalized to the house keeping gene GAPDH (assay ID: Mm99999915_g1). Thermal cycling was initiated with a denaturation step of $10 \mathrm{~min}$ at
$95{ }^{\circ} \mathrm{C}$, followed by 45 cycles of $15 \mathrm{~s}$ at $95{ }^{\circ} \mathrm{C}$ and $90 \mathrm{~s}$ at $60{ }^{\circ} \mathrm{C}$. Each sample was analyzed in triplicate alongside with negative control. The data were obtained as cycle threshold $(\mathrm{Ct})$ values and comparative gene expression analysis was performed using and the $2^{-\Delta \Delta \mathrm{Ct}}$ relative quantification method.

In silico analysis was performed to establish the relationship between miRNA and FOS and FTO. Three different miRNA prediction softwares including MiRanda (https ://www.microrna.org/microrna), Target Scan 7.2 (https:// www.targetscan.org/vert_72) and miRwalk (https://zmf. umm.uni-heidelberg.de/apps/zmf/mirwalk2) were used to screen the miRNA-binding possibility against FOS and FTO 3'UTR. Based on their binding potential against FOS and FTO and functional involvement in hypothalamus, a total of twelve miRNAs were selected (listed in Table 1). All the qPCR experiments for miRNA expression were performed in 96-well plates with QuantStudio3 Realtime PCR (Applied Biosystems) using SYBR green (Qiagen). The list of primers used for miRNA expressions is presented in Table 1. The expression of individual miRNA was normalized against the expression of U6SnRNA. Amplification reactions were performed in a volume of $10 \mu \mathrm{L}$ containing cDNA, SYBR green master mix, corresponding primer assays (forward primer) and a universal primer (reverse primer) for each miRNA. Thermal cycling was initiated with a denaturation step of $10 \mathrm{~min}$ at $95^{\circ} \mathrm{C}$, followed by 40 cycles of $15 \mathrm{~s}$ at $94{ }^{\circ} \mathrm{C}$ and $30 \mathrm{~s}$ at $55^{\circ} \mathrm{C}$ followed by $30 \mathrm{~s} 72{ }^{\circ} \mathrm{C}$. Each sample was analyzed in triplicate alongside negative control. The data were obtained as cycle threshold $(\mathrm{Ct})$ values and comparative gene expression analysis was performed using and the $2^{-\Delta \Delta \mathrm{Ct}}$ relative quantification method.

Table 1 MicroRNA primer sequence

\begin{tabular}{lll}
\hline S. no. & miRNA & miRNA primer sequence $\left(5^{\prime}-3^{\prime}\right)$ \\
\hline 1 & mmu-miR-7a-5p & TGGAAGACTAGTGATTTTGTTGT \\
2 & mmu-miR-7b-5p & TGGAAGACTTGTGATTTTGTTG \\
3 & mmu-miR-9-5p For & TCTTTGGTTATCTAGCTGTATGA \\
4 & mmu-miR-29b-3p & TAGCACCATTTGAAATCAGTG \\
5 & mmu-miR-29c-3p & TAGCACCATTTGAAATCGG \\
6 & mmu-miR-30a-5p & TGTAAACATCCTCGACTGGAAG \\
7 & mmu-miR-30b-5p & TGTAAACATCCTACACTCAGC \\
8 & mmu-miR-30c-5p & TGTAAACATCCTACACTCTCAG \\
9 & mmu-miR-338-3p & TCCAGCATCAGTGATTTTGTTG \\
10 & mmu-miR-873a-5p & GCAGGAACTTGTGAGTCTCCT \\
11 & mmu-miR-101b-3p & GTACAGTACTGTGATAGCT \\
12 & mmu-miR-181a-5p & AACATTCAACGCTGTCGGTGAGT \\
13 & mmu-U6SnRNA & TGGCCCCTGCGCAAGGATG \\
\hline
\end{tabular}




\section{Protein extraction and western blotting}

For western blot analysis, $0.5 \times 10^{6}$ cells were seeded in $100 \mathrm{~mm}$ cell culture dish (ThermoFisher Scientific) with different glucose concentrations and were grown for $72 \mathrm{~h}$. Cells were pelleted and washed with ice cold PBS. Protein lysates were prepared by performing cell lysis using M-PER mammalian protein extraction reagent (ThermoFisher Scientific) containing protease inhibitors (ThermoFisher Scientific). Total protein concentrations were measured with standard Bradford method (Bio-Rad). Lysates containing $50 \mu \mathrm{g}$ of total protein were separated on $12 \%$ sodium dodecyl sulfate-polyacrylamide gel electrophoresis (SDS-PAGE) and blotted onto nitrocellulose membrane (Biorad) for $30 \mathrm{~min}$ at $30 \mathrm{~V}$ in a transfer buffer containing Tris-base and glycine. The membranes were blocked with 5\% skim milk prepared in Tris-buffered saline with $0.1 \%$ of Tween 20 (TBST) for $1 \mathrm{~h}$ and then incubated with primary antibodies against FOS (1:1000 dilutions; Abcam), FTO (1:1000 dilutions; Abcam) and $\beta$-actin (1:5000 dilutions; Sigma-Aldrich) at $4{ }^{\circ} \mathrm{C}$ overnight. The membranes were then washed with TBST and incubated with horseradish peroxidase-linked secondary antibody at room temperature for $1 \mathrm{~h}$. After TBST washing, the membranes were visualized by clarity western ECL substrate (Bio-Rad) according to the manufacturer's procedure. The intensity of bands was quantified using image $\mathrm{J}$ software.

\section{MiRNA transfection}

$1 \times 10^{5}$ cells were cultured in $60 \mathrm{~mm}$ cell culture plates (Sigma). Four different hypoglycemic conditions were established by seeding the cells in DMEM containing $2000 \mathrm{mg} / \mathrm{L}$, $900 \mathrm{mg} / \mathrm{L}, 500 \mathrm{mg} / \mathrm{L}$ and $200 \mathrm{mg} / \mathrm{L}$ of glucose along with normo-glycemic condition $(4500 \mathrm{mg} / \mathrm{L})$. Next day cells were transfected using $60 \mathrm{nM}$ miR-9-5p mimic (Thermo-scientific \# 4464066), antimiR-9 (Thermo-scientific \# 4464084) and negative control separately with the help of lipofactamine RNAimax (Thermo-scientific). After $24 \mathrm{~h}$, transfected media were replaced by DMEM media containing respective hypoglycemic condition and kept for $72 \mathrm{~h}$. After incubation, cells were pelleted and washed in ice cold PBS. Protein lysates were prepared by performing cell lysis using M-PER mammalian protein extraction reagent (Thermo scientific) containing protease inhibitors (Thermo scientific) and further continued for western blot analysis.

\section{Statistical analysis}

Data are expressed as \pm standard deviation (SD). All statistical analyses were performed using GraphPad Prism 7 for windows (GraphPad Software, CA, USA). Statistical comparisons were performed by one-way analysis of variance
(ANOVA) followed by Dunnett's post hoc test for comparing different groups to control $(4500 \mathrm{mg} / \mathrm{L}$ glucose) or the Tukey's correction for multiple comparison. Probability values of $p<0.05$ were considered statistically significant.

\section{Results}

\section{Effects of low glucose conditions on the viability of hypothalamic neurons}

The first group of experiments was designed to examine the viability of the hypothalamic neurons in response to different conditions of low glucose concentrations. As shown in Fig. 1, the viability of the hypothalamic neurons was decreased significantly due to low glucose conditions and this response was dose dependent $(p<0.05)$. As the concentrations of glucose $(2000 \mathrm{mg} / \mathrm{L}, 900 \mathrm{mg} / \mathrm{L}$, $500 \mathrm{mg} / \mathrm{L}$ and $200 \mathrm{mg} / \mathrm{L}$ ) were reduced, the survival of the neurons was affected, adversely. The duration of exposure to low levels of glucose did not influence the survival of the neurons, significantly. The outcomes of cell viability during 24,48 and $72 \mathrm{~h}$ exposure to low levels of glucose were comparable (Fig. 1). However, the comparison of neurons survival between 24 and $72 \mathrm{~h}$ under two conditions with low glucose concentration $(900 \mathrm{mg} / \mathrm{L}$ and $500 \mathrm{mg} / \mathrm{L}$ of glucose), in particular, has shown a significant reduction in the survival rate which was duration dependent $(p<0.05)$.

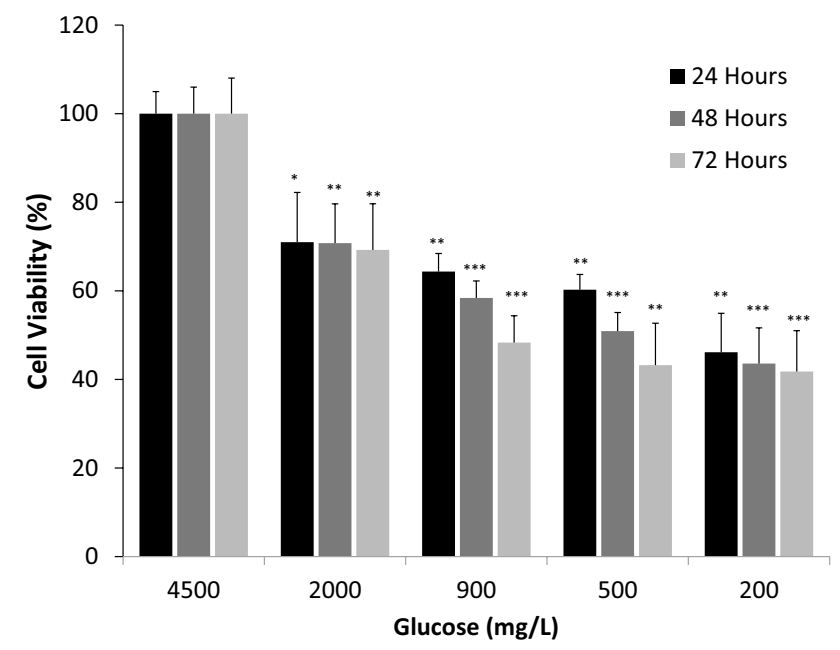

Fig. 1 Viability of hypothalamic neurons after exposure to various concentration of glucose. Concentrations of glucose $(\mathrm{mg} / \mathrm{L})$ were $4500,2000,900,500$ and 200 and the duration of exposure was 24 , 48 , and $72 \mathrm{~h}$. Data are presented as the mean $\pm \mathrm{SD}(n=6, * P<0.05$; $* * P<0.01, * * * P<0.001$, compared to normoglycemia condition of $4500 \mathrm{mg} / \mathrm{L}$ glucose) 


\section{Effects of low glucose conditions on the cell death of hypothalamic neurons}

As shown in Fig. 2a, the apoptotic rate was closely related to the glucose concentration and there was a significant shift toward more apoptosis (early and late) in response to conditions with low glucose concentration. This response was dose dependent and the condensation and fragmentation of the nuclei indicating the apoptotic nature, significantly increased as the glucose concentration decreased. Although reduced concentration of glucose produced gradual increase in the apoptotic rate, the two lowest concentrations, 500 and $200 \mathrm{mg} / \mathrm{L}$ of glucose, produced the most significant effects (Fig. 2b).

\section{FOS and FTO mRNA expression in the embryonic hypothalamic neurons in response to low glucose conditions}

Expression of FOS was increased significantly in response to low glucose concentrations and this response was dose dependent ( $p<0.05$; Fig. 3). FOS expression increased as the concentration of glucose decreased indicating an inverse relationship between expression of FOS and low glucose concentration. In contrast, the expression of FTO decreased as the concentrations of glucose were decreased, showing a

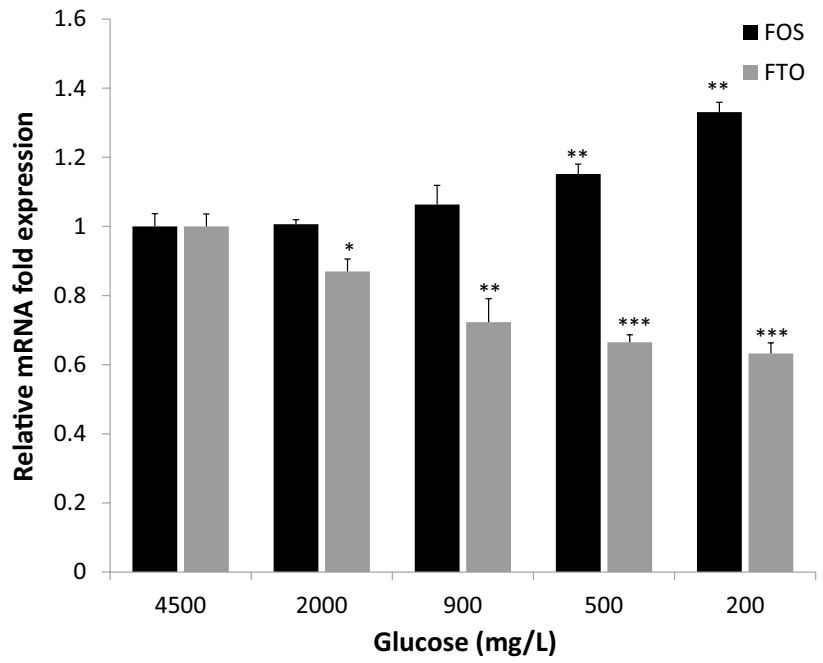

Fig. 3 Expression of FOS and FTO mRNA transcripts in the embryonic hypothalamic neurons. Fold difference in FOS (Black Bars) and FTO (Gray Bars) mRNA levels in response to low glucose conditions (2000, 900, 500 and $200 \mathrm{mg} / \mathrm{L}$ ) was compared with mRNA levels under normoglycemic condition $(4500 \mathrm{mg} / \mathrm{L})$ as the reference $=1$, as determined by quantitative reverse transcription-polymerase chain reaction. Error bars represent the standard deviations $(n=3$, $* P<0.05, * * P<0.01, * * * P<0.001)$
A

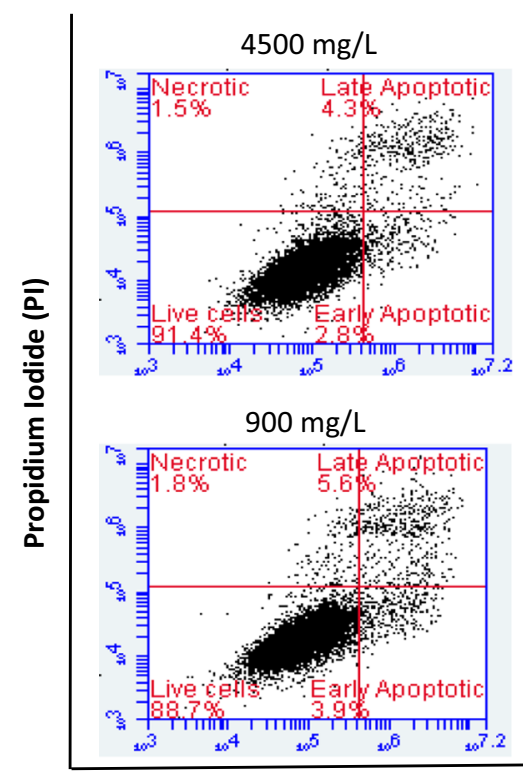

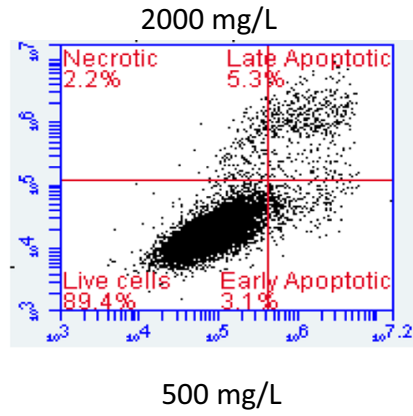

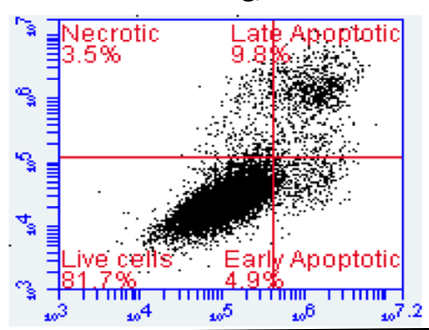

Annexin-V
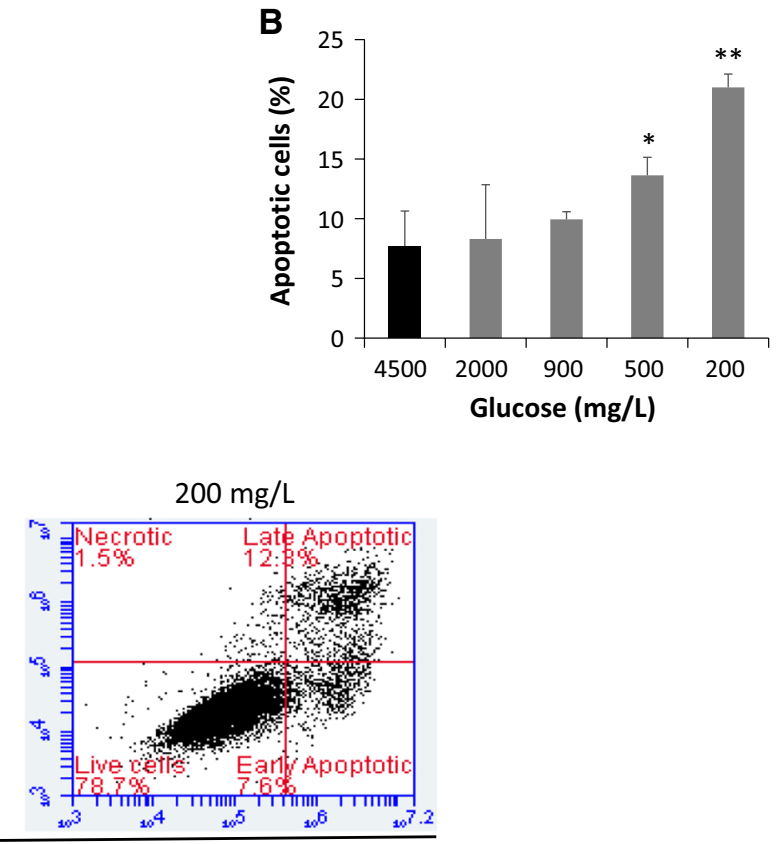

.

Fig. 2 Low glucose condition-induced apoptosis in hypothalamic neurons evaluated by flow cytometry. a An increase in the early and late apoptosis of the neurons in a dose-dependent fashion. $\mathbf{b}$ The rate of apoptosis in the hypothalamic neurons in response to low glucose conditions (2000-200 mg/L of glucose) 
direct proportional relationship between expression of FTO and glucose concentrations (Fig. 3).

\section{FOS and FTO protein expression in the embryonic hypothalamic neurons in response to low glucose conditions}

As shown in Fig. 4, quantitation of FOS and FTO protein using Western Blotting revealed that FOS expression was increased in response to low glucose conditions $(2000 \mathrm{mg} / \mathrm{L}$, $900 \mathrm{mg} / \mathrm{L}, 500 \mathrm{mg} / \mathrm{L}$ and $200 \mathrm{mg} / \mathrm{L}$ of glucose) as shown by blots probed with FOS antibody (Fig. 4a, b). In contrast, FTO expression was decreased in response to the same low glucose conditions (Fig. 4a, b). Changes in the expression of both FOS and FTO in response low glucose conditions were statistically significant $(p<0.05)$.

\section{Differential expression of miRNAs in the embryonic hypothalamic neurons in response to low glucose conditions}

To investigate the involvement of miRNAs in regulation of FOS and FTO expression under the low glucose conditions, in silico analysis was performed using miRanda, Target Scan 7.2 and miRwalk databases. The analysis has shown differential expression of more than 1000 miRNAs with
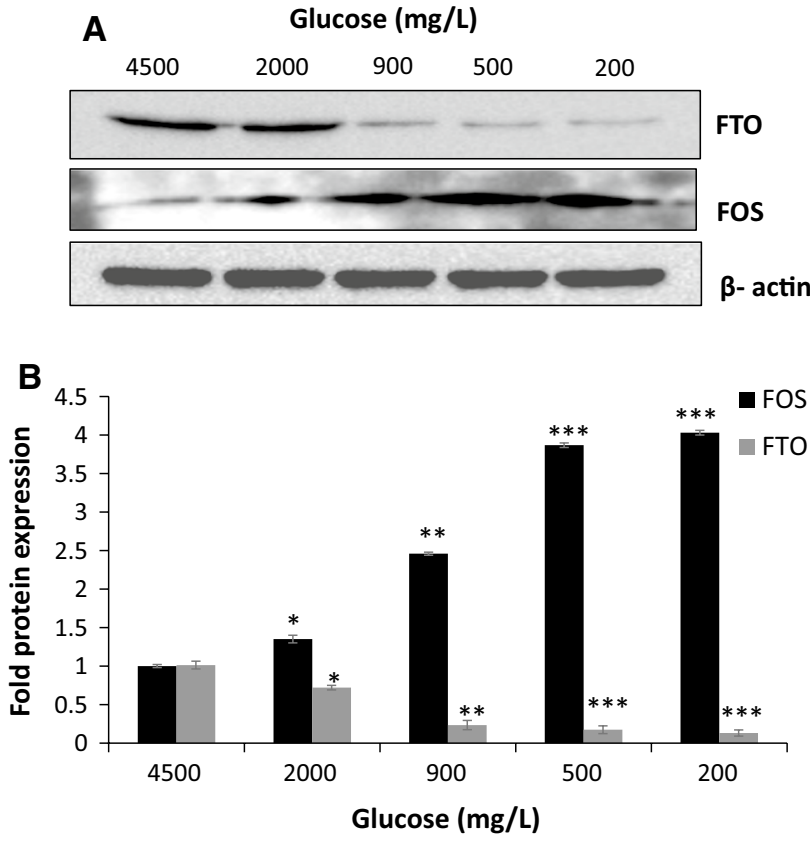

Fig. 4 Quantitation of Western blot FOS and FTO protein expression in response to low glucose conditions. a Representative Western blot of FOS and FTO in response to different glucose conditions (2000, 900, 500 and $200 \mathrm{mg} / \mathrm{L}$ ). b Protein levels of FOS (Black Bars) and FTO (Gray Bars) were corrected to actin expression $(* P<0.05$; $* * P<0.01, * * * P<0.001)$ binding potency against FOS and FTO coding sequence and untranslated region (UTR). However, only twelve potential candidates' miRNAs were identified based on their binding potential against FOS and FTO with a direct involvement in the hypothalamus. The latter includes: miR-7a, miR-7b, miR-9, miR-29b, miR-29c, miR-30a, miR-30b, miR-30c, miR-101b-3p, miR 181a-5p, miR-378-3p and miR-873-5p. As shown in Fig. 5a, the expression of miR-7a, miR-7b was significantly reduced in response to low glucose concentrations of 2000, 900, 500 and $200 \mathrm{mg} / \mathrm{L}(p<0.05 ; p<$ 0.01 ). Expression of miR-29b and miR-29c was significantly reduced in response to low glucose concentrations of 2000, 900, 500 and $200 \mathrm{mg} / \mathrm{L}$ (Fig. 5b; $p<0.05 ; p<0.01 ; p$ $<0.001$ ). Expression of miR-30a, miR-30b, miR-30c was significantly reduced in response to low glucose concentrations of 2000, 900, 500 and $200 \mathrm{mg} / \mathrm{L}$ (Fig. $5 \mathrm{c} ; p<0.05 ; p$ $<0.01 ; p<0.001)$. Similarly, the expression of miR-9 was significantly reduced in response to low glucose concentrations of 2000, 900, 500 and $200 \mathrm{mg} / \mathrm{L}$ (Fig. $5 \mathrm{~d} ; p<0.01$ ). As shown in Fig. 5e, miR- 378-3p was significantly reduced in response to low glucose concentrations of 900,500 and $200 \mathrm{mg} / \mathrm{L}(p<0.05)$. In addition, miR-873-5p was reduced in response to low glucose concentrations of 2000, 900, 500 and $200 \mathrm{mg} / \mathrm{L}$ (Fig. 5f; $p<0.05 ; p<0.01$ ). However, no significant change was observed in the expression level of miR-101b-3p and miR-181-5p in response to low glucose conditions (Fig. $5 \mathrm{~g} ; p>0.05$ ).

\section{Regulatory effects of miR-9 on the expression of FOS under low glucose conditions}

As mentioned above, twelve potential candidates' miRNAs were identified based on their binding potential against FOS. Out of these twelve miRNAs, miRNA-9 expression was significantly reduced in response to all conditions of low glucose conditions; therefore, miRNA-9 was chosen as a candidate to do further experiments to assess the role of miRNA-9 in regulation of FOS expression under low glucose concentrations. The results of these experiments have shown that miRNA-9 negatively regulates the expression of FOS. As shown in Fig. 6, FOS expression was decreased in response to reduction of glucose concentration in presence of miR-9 mimic. This response seems to be dependent on the concentration of glucose and, at $2000 \mathrm{mg} / \mathrm{L}$ concentration of glucose, the reduction of FOS in the presence of miR-9 mimic was $27 \%$ (Fig. 6b), whereas glucose concentration of $200 \mathrm{mg} / \mathrm{L}$ resulted in almost 90\% reduction (Fig. 6e) in FOS expression in the presence of miR-9 mimic. More importantly, the presence of AntimiR-9 reversed these inhibitory effects of miR-9 mimic and increased the expression of FOS, significantly. This effect seems also to be dependent on the glucose concentration; as in the $2000 \mathrm{mg} / \mathrm{L}$ concentration of glucose, the FOS expression increased about 

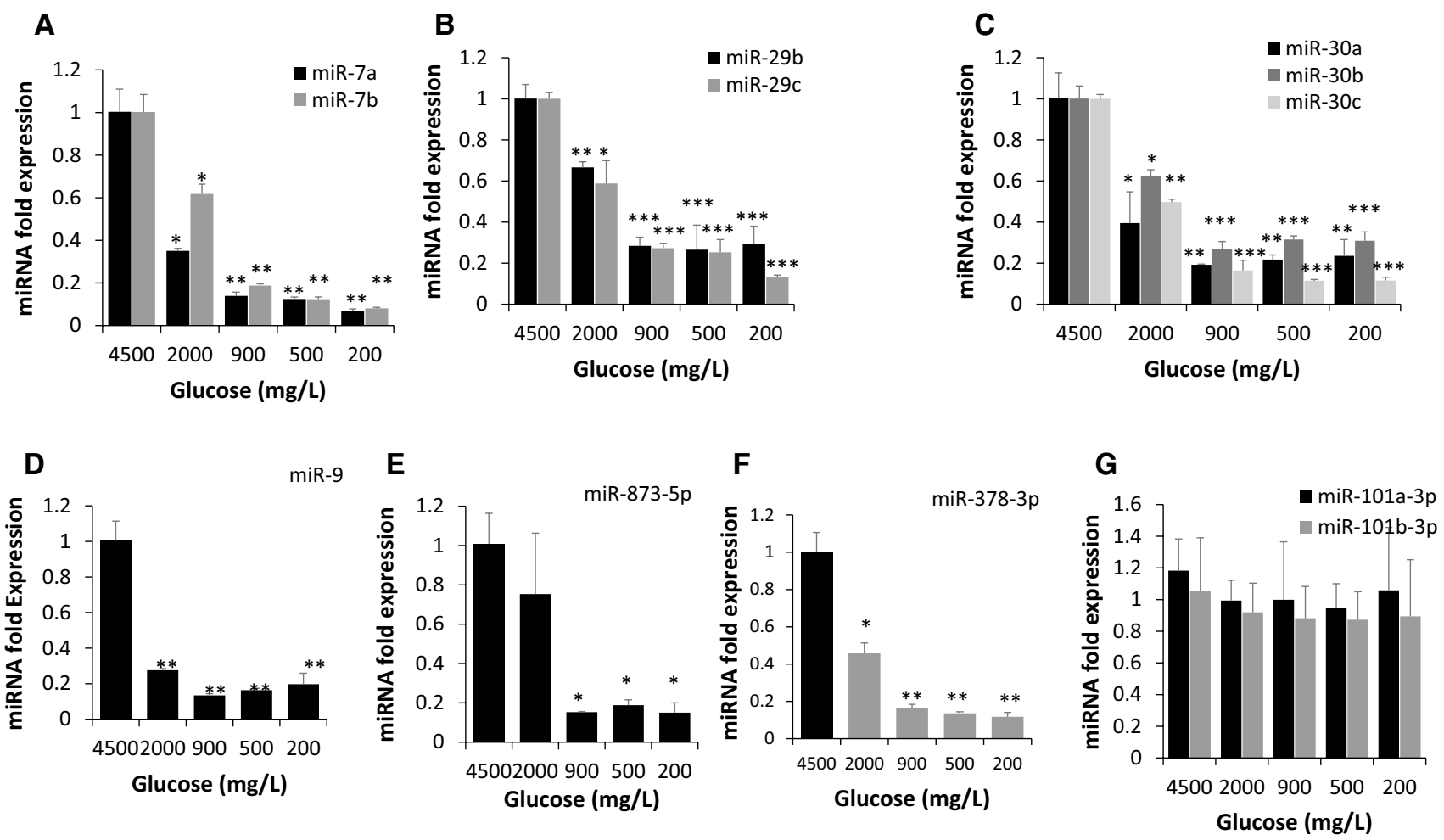

Fig. 5 Differential expression of miRNAs in hypothalamic neurons in response to low glucose conditions. a Expression of miR-7a (black bar) and $-7 b$ (gray bar) was significantly reduced in response to low glucose conditions of 2000, 900, 500 and $200 \mathrm{mg} / \mathrm{L}$ ( $* p<0.05$; $* * p<0.01$ ). b Expression of miR-29b (Black Bar) and miR-29c (gray bar) was significantly reduced in response to low glucose conditions of 2000, 900, 500 and $200 \mathrm{mg} / \mathrm{L}(* p<0.05 ; * * p<0.01$; $* * * p<0.001$ ). c Expression of miR-30a (black bar), -30b (gray bar), -30 c (white bar) was significantly reduced in response to low glucose

conditions of $2000,900,500$ and $200 \mathrm{mg} / \mathrm{L}(* p<0.05 ; * * p<0.01$; $* * * p<0.001)$. d Expression of miR-9 was significantly reduced in response to low glucose conditions of $2000,900,500$ and $200 \mathrm{mg} / \mathrm{L}$ $(* p<0.01)$. e, f Expression of miR-378-3p and miR-873-5p was significantly reduced in response to low glucose conditions of 900,500 and $200 \mathrm{mg} / \mathrm{L}(* p<0.05 ; * * p<0.01 ; * * * p<0.001)$. g No significant differences were found in the expression of miR-101a-3p and miR$101 b-3 p$ under different glycemic conditions $(p>0.05)$
A

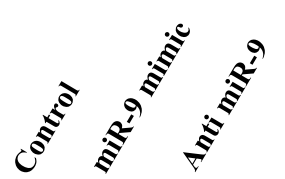

$62 \mathrm{KD}$
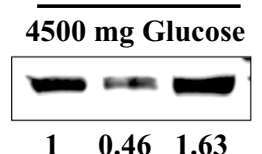

$42 \mathrm{KD}$

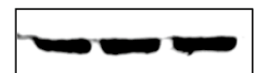

B

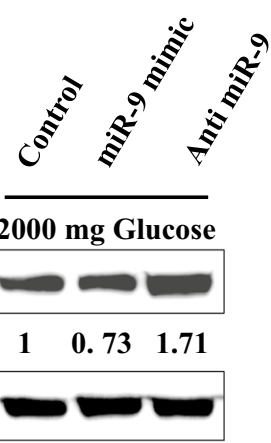

C

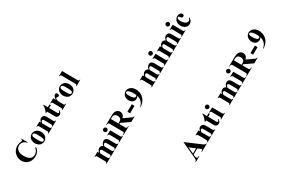

D

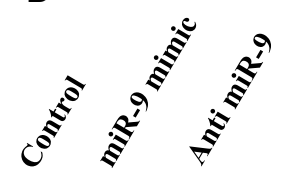

E

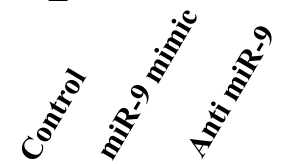

Fig. 6 Expression of FOS in response to miR-9 mimic and antimiR-9 using western blot. Representative Western blot of FOS in response to different low glucose conditions in the presence of miR-9 mimic and antimiR-9 (a $4500 \mathrm{mg} / \mathrm{L}$ glucose, b $2000 \mathrm{mg} / \mathrm{L}$ glucose, $\mathbf{c}$ $900 \mathrm{mg} / \mathrm{L}$ glucose, $\mathbf{d} 500 \mathrm{mg} / \mathrm{L}$ glucose and e $200 \mathrm{mg} / \mathrm{L}$ glucose) 
$70 \%$ (Fig. 6b) in the presence of AntimiR-9 and increased to $120 \%$ (Fig. 6e) when the glucose concentration reduced to $200 \mathrm{mg} / \mathrm{L}$.

\section{Discussion}

Hypoglycemia-induced neuronal injury represents a significant challenge to achieve optimal glycemic control in patients with type $1 \mathrm{DM}$. More importantly, recurrence of hypoglycemic episodes leads to impairment of critical reflexes that are naturally designed to restore normoglycemia and this, in turn, leads to HAAF [13]. Early detection of the latter will improve glycemic control and reduce the serious complications of HAAF. We hypothesized that miRNAs that are involved in regulation of key hypothalamic proteins, such as FOS and FTO, can be clinically relevant biomarkers.

Several neurophysiological studies have investigated different aspects of hypoglycemia and the homeostatic responses to this condition such as CCR [21, 22]. However, none of the previous reports has examined the molecular signature of hypoglycemia in embryonic neurons within the hypothalamus. In addition, the associated effects of hypoglycemia on expression of miRNAs and the hypothalamic regulatory proteins are yet to be reported. The hypothalamus is a heterogeneous region and, therefore, the best model to study the electrophysiological properties of this region is the in vivo model. However, to elucidate the underlying molecular mechanisms of different hypothalamic processes, cell lines represent the most appropriate method and that is why many researchers have turned towards cell lines models [31]. Although primary hypothalamic cultures are heterogeneous and mimic the nature of the hypothalamus in the living brain, they are difficult to maintain and lack viable peptide-secreting neurons. In contrast, embryonic mouse hypothalamus cells (mHypoE-N39) are unlimited homogenous neurons that can be manipulated by various molecular techniques to enrich the molecular aspects of the hypothalamic neurons under different conditions [32]. The latter was the main reason to use these hypothalamic cells in the present study.

The findings of the present study have shown that the survival of the EHNs is dependent on the availability of glucose and, therefore, the hypoglycemic conditions produced a dose-dependent neuronal death. In agreement with these findings, previous reports have shown that hypoglycemia led to more than $90 \%$ neuronal death in cortical neuron cultures [33]. This effect was dependent on the time of exposure to glucose where $7 \mathrm{~h}$ of glucose deprivation caused more significant neuronal death compared to $3 \mathrm{~h}$ [30]. The present study examined the effects of both exposure time and low concentrations of glucose on the survival of the EHNs. Compared to the duration of hypoglycemia, the effects of decreased glucose concentrations seem to be more powerful and consistent. In addition to the different approaches that were used to induce hypoglycemia, the fact that different neurons in the brain possess different thresholds to respond to hypoglycemia explains the variation in the outcomes between the previous and present studies.

Previous electrophysiological studies have used in vitro approach to examine the glucose-sensing behavior within the hypothalamus and this contributed significantly to a better understanding of different aspects of counter-regulation of glycemia and feeding behavior $[34,35]$. Most of these studies used a single low dose of glucose concentration to induce hypoglycemia in hypothalamic neurons and the assessment of the neural survival was not the main focus of these studies. On the other hand, the present study has used the approach of gradual reduction of glucose using four doses of low glucose to induce hypoglycemia and also assessed the survival rate of the neurons in more comprehensive manner. It is noteworthy that with recent advancements, the availability of no-glucose media provided an opportunity to prepare the culture medium with lower levels of glucose concentrations and this helped in establishing a better approach to examine the direct effects of hypoglycemia on neural survival [36].

Upon further investigation of the nature of the neuronal death in response to hypoglycemia, findings have shown that the latter induced apoptosis in the EHNs and this was particularly significant at the two lowest concentrations of glucose. These findings were supported by previous in vivo reports which have demonstrated that hypoglycemic rats (1 bout $39 \pm 5 ; 3$ bouts $37 \pm 4$ ) had more apoptotic cells within the arcuate hypothalamic nucleus cells compared to euglycemic control rats $(13 \pm 3 ; p=0.001)$ [37]. These apoptotic effects of hypoglycemia were observed after a single bout of hypoglycemia supporting the hypothesis that apoptosis is the underlying cause of neuronal death in the hypothalamus in response to hypoglycemia.

Although the present study has confirmed the neuronal death in response to direct hypoglycemia, an exceptional phenomenon was observed which is an inverse relationship between FOS expression and glucose concentrations in the EHNs. Previously, changes of FOS expression in response to hypoglycemia were used to determine the location of the neurons that are functionally related to hypoglycemia without in-depth studies about the molecular aspects of FOS expression during the process of neuronal death [38-40]. Although there was no consensus in the outcomes of these previous studies given the heterogeneity of the hypothalamus, the majority suggested that low glucose concentrations lead to reduction in FOS expression [22]. In contrast, the present study has demonstrated that hypoglycemia causes 
an increase in expression of FOS despite the death of EHNs, suggesting a protective role of FOS against hypoglycemia. This may reflect the original function of FOS since the present experiments were conducted in embryonic neurons whereas all previous experiments were performed in adult neurons. On the other hand, two categories of neurons were previously suggested based on the behavior of glucose sensing: (i) glucose responsive (GR) excited neurons and (ii) glucose-sensitive (GS) inhibited neurons [41]. In the latter, it is natural that lowering glucose increases FOS expression, cytosolic $\mathrm{Ca}^{2+}$ and membrane depolarization and these parameters were used as markers for neuronal excitation of GS neurons in the lateral hypothalamic areas $[42,43]$ and the arcuate nucleus $[44,45]$. To consider these previous findings as alternative explanation for our present studies, we believe that further studies need to be conducted to investigate the electrophysiological features of EHNs under hypoglycemic conditions.

Simultaneously, FTO expression was assessed in the same hypoglycemic environment. Interestingly, reduced hypothalamic FTO expression was reported in response to hypoglycemia in a dose-dependent fashion. In agreement with this finding, a study by Poritsano et al. has shown that fasting negatively influences FTO mRNA levels and the number of FTO-immunoreactive cells in the hypothalamus. These outcomes were reversed in response to administration of glucose [2]. In the light of these findings and along with the present outcomes, we can confirm the regulatory role of hypothalamic FTO in the mechanisms of hypoglycemia in adult and embryonic neurons within the hypothalamus. In addition, an interesting relationship between variants in FTO and obesity has been suggested emphasizing the role of FTO in central regulation of several metabolic processes [46]. This was further supported by the findings that mouse models with the absence of FTO exhibited reduced body weight whereas overexpression of FTO resulted in an increase in body weight [47, 48]. Although FTO is expressed in various tissues, its level is particularly high in the hypothalamus and this suggests that the hypothalamic FTO plays an important role in glucose metabolism as well as in obesity. Given that the obesity has a strong heritable component, further genetic studies have shown that FTO encodes for an enzyme able to remove methyl groups from DNA and RNA nucleotides in vitro and human studies, emphasizing the involvement of epigenetic mechanisms in obesity $[49,50]$. Taking into account the findings of the previous and present studies, we can suggest an association between hypothalamic FTO and epigenetic alterations that may involve in hypoglycemia and obesity; however, further studies are required to support this suggestion.

Recently, the involvement of miRNAs in regulation of hypothalamic functions has received much attention. Several genes within the hypothalamus are regulated by miRNAs and, therefore, it is not surprising that these non-coding RNAs are potential biomarkers and modulators for various diseases and future therapeutic targets [14, 51-53]. Given the holistic role of hypothalamus in regulation of several physiological functions including food intake and body weight, previous reports have investigated the involvement of miRNAs in these functions [14]. However, the involvement of miRNAs in detection or responding to glycemic changes is considered as a novel field of research. In the present study, screening for miRNAs has shown that more than 1000 miRNAs are differentially expressed in the hypothalamus. Nevertheless, overlapping binding sites for twelve miRNAs were identified in FOS and FTO. It is noteworthy that there was an overlap in the miRNAs between FTO and FOS despite the fact that both had differential responses to hypoglycemia. This highlights an important interrelationship between central integration of food intake, obesity and glycemic control.

In addition, these inhibitory regulators were gradually suppressed by reduction in glucose concentrations suggesting that hypoglycemia may have negative effect on the expression of these miRNAs. Taking into account this finding, we can suggest that the noticeable increase in FOS expression in response to hypoglycemia may result from the inhibition of miRNAs expression. This suggestion was strongly supported by the finding that miR-9 negatively regulates the expression of FOS and this effect was dependent on the concentration of glucose. As the latter decreases, the miR-9 mimic produces more significant reduction in the expression of FOS and AntimiR-9 leads to pronounced increase in expression of FOS. We believe that the relationship between hypothalamic miRNAs and regulatory proteins, FOS and FTO, builds a strong potential for identification of novel biomarkers for HAAF.

\section{Conclusion}

The results of the present study demonstrated that optimal glycemic level is crucial for survival of EHNs. FOS expression was significantly increased in response to hypoglycemia suggesting a protective role in this condition. Twelve miRNAs that are related to both FOS and FTO were identified and the expression of ten of them was reduced significantly in response to hypoglycemia. This interrelationship between the hypothalamic miRNAs and the regulatory protein, FOS and FTO, in response to hypoglycemia facilitates an opportunity to identify potential biomarkers and novel therapeutic targets for HAAF.

Acknowledgements The authors would like to thank Dr. Abbas Jarrahi (University of Sharjah, Sharjah, UAE) for his help in the initial preparation of the experimental protocols. Also, we would like to thank Mr. 
Tahir Maqbool (Cedarlane, Ontario, Canada) for his technical help in maintaining the hypothalamic cells.

Author contributions BMM developed and designed the study; AM, AS, DM performed the experiments and analyzed the data; BMM and JT interpreted the results of experiments. All authors revised, edited and approved the final version of the manuscript.

Funding The study was supported by Grants from LABCO (UOSLABCO2016) and Al Jalila Foundation (AJF2018082).

\section{Compliance with ethical standards}

Conflict of interest The authors have no personal, financial or nonfinancial conflict of interest.

Open Access This article is distributed under the terms of the Creative Commons Attribution 4.0 International License (http://creativeco mmons.org/licenses/by/4.0/), which permits unrestricted use, distribution, and reproduction in any medium, provided you give appropriate credit to the original author(s) and the source, provide a link to the Creative Commons license, and indicate if changes were made.

\section{References}

1. Dalvi PS, Nazarians-Armavil A, Tung S, Belsham D (2011) Immortalized neurons for the study of hypothalamic function. Am J Physiol Regul Integr Comp Physiol 300(5):R1030-R1052

2. Poritsanos NJ, Lew PS, Fischer J, Mobbs CV, Nagy JI, Wong D et al (2011) Impaired hypothalamic Fto expression in response to fasting and glucose in obese mice. Nutr Diabetes 1:e19

3. Saper CB, Lowell BB (2014) The hypothalamus. Curr Biol 24(23):R1111-R1116

4. Moraes JC, Coope A, Morari J, Cintra DE, Roman EA, Pauli JR et al (2009) High-fat diet induces apoptosis of hypothalamic neurons. PLoS ONE 4:e5045

5. Zhang X, Zhang G, Zhang H, Karin M, Bai H, Cai D (2008) Hypothalamic IKK $\beta / \mathrm{NF}-\kappa \mathrm{B}$ and ER stress link overnutrition to energy imbalance and obesity. Cell 135(1):61-73

6. Pinto S, Roseberry AG, Liu H, Diano S, Shanabrough M, Cai X et al (2004) Rapid rewiring of arcuate nucleus feeding circuits by leptin. Science 304:110-115

7. Page KA, Arora J, Qiu M (2009) Small decrements in systemic glucose provoke increases in hypothalamic blood flow prior to the release of counterregulatory hormones. Diabetes 58:448-452

8. Otlivanchik O, Le Foll C, Levin BE (2015) erifornical hypothalamic orexin and serotonin modulate the counterregulatory response to hypoglycemic and glucoprivic stimuli. Diabetes 64:226-235

9. Korim WS, Bou Farah L, McMullan S, Verberne AJ (2014) Orexinergic activation of medullary premotor neurons modulates the adrenal sympathoexcitation to hypothalamic glucoprivation. Diabetes 63:1895-1906

10. Cryer PE (2001) Hypoglycemia-associated autonomic failure in diabetes. Am J Physiol Endocrinol Metab 281:E1115-E1121

11. Frier BM, Schernthaner G, Heller SR (2011) Hypoglycemia and cardiovascular risks. Diabetes Care 34:S132-S137

12. Geddes J, Schopman JE, Zammitt NN, Frier BM (2008) Prevalence of impaired awareness of hypoglycaemia in adults with Type 1 diabetes. Diabetes Med 25(4):501-504

13. Cryer PE (2010) Hypoglycemia in type 1 diabetes mellitus. Endocrinol Metab Clin N Am 39:641-654
14. Meister B, Herzer S, Silahtaroglu A (2013) MicroRNAs in the hypothalamus. Neuroendocrinology 98(4):243-253

15. Benoit C, Ould-Hamouda H, Crepin D, Gertler A, Amar L, Taouis MJ (2013) Early leptin blockade predisposes fat-fed rats to overweight and modifies hypothalamic microRNAs. J Endocrinol 218:35-47

16. Schneeberger M, Altirriba J, García A, Esteban Y, Castaño C, García-Lavandeira M et al (2012) Deletion of miRNA processing enzyme Dicer in POMC-expressing cells leads to pituitary dysfunction, neurodegeneration and development of obesity. Mol Metab 2(2):74-85

17. Lee H-J, Palkovits M, Young WS (2006) miR-7b, a microRNA up-regulated in the hypothalamus after chronic hyperosmolar stimulation, inhibits Fos translation. Proc Natl Acad Sci USA 103:15669-15674

18. Cai XJ, Widdowson PS, Harrold J, Wilson S, Buckingham RE, Arch JR et al (1999) Hypothalamic orexin expression: modulation by blood glucose and feeding. Diabetes 48(11):2132-2137

19. Moriguchi T, Sakurai T, Nambu T, Yanagisawa M, Goto K (1999) Neurons containing orexin in the lateral hypothalamic area of the adult rat brain are activated by insulin-induced acute hypoglycemia. Neurosci Lett 264:101-104

20. Korim W, Llewellyn-Smith I, Verberne A (2016) Activation of medulla-projecting perifornical neurons modulates the adrenal sympathetic response to hypoglycemia: involvement of orexin type 2 (OX2-R) receptors. Endocrinology 157:810-819

21. Al-Noori S, Sanders NM, Taborsky GJ, Wilkinson CW, Zavosh A, West C et al (2008) Recurrent hypoglycemia alters hypothalamic expression of the regulatory proteins FosB and synaptophysin. Am J Physiol Regul Integr Comp Physiol 295(5):R1446-R1454

22. Foster NN, Azam S, Watts AG (2016) Rapid-onset hypoglycemia suppresses Fos expression in discrete parts of the ventromedial nucleus of the hypothalamus. Am J Physiol Regul Integr Comp Physiol 310(11):R1177-R1185

23. Levin BE, Routh VH, Kang L, Sanders NM, Dunn-Meynell AA (2004) Neuronal glucosensing: what do we know after 50 years? Diabetes 53(10):2521-25218

24. Xu G, Sima A (2001) Altered immediate early gene expression in injured diabetic nerve: implications in regeneration. J Neuropathol Exp Neurol 60(10):972-983

25. Ness J, Harvey C, Washington C, Roth K, Carroll S, Zhang J (2008) Differential activation of c-Fos and caspase- 3 in hippocampal neuron subpopulations following neonatal hypoxia-ischemia. J Neurosci Res 86(5):1115-1124

26. Olson BR, Freilino M, Hoffman GE, Stricker EM, Sved AF, Verbalis JG (1993) c-Fos expression in rat brain and brainstem nuclei in response to treatments that alter food intake and gastric motility. Mol Cell Neurosci 4(1):93-106

27. Gerken T, Girard CA, Tung YC, Webby CJ, Saudek V, Hewitson KS et al (2007) The obesity-associated FTO gene encodes a 2-oxoglutarate-dependent nucleic acid demethylase. Science 318(5855):1469-1472

28. Olszewski PK, Fredriksson R, Olszewska AM, Stephansson O, Alsiö J, Radomska KJ et al (2009) Hypothalamic FTO is associated with the regulation of energy intake not feeding reward. BMC Neurosci 10(129):1-12

29. Gerken T, Girard CA, Tung YC, Webby CJ, Saudek V, Hewitson KS et al (2008) Novel genetic variant in FTO influences insulin levels and insulin resistance in severely obese children and adolescents. Int J Obes Lond 32(11):1730-1735

30. Horikoshi M, Hara K, Ito C, Shojima N, Nagai R, Ueki K et al (2007) Variations in the HHEX gene are associated with increased risk of type 2 diabetes in the Japanese population. Diabetologia 50(12):2461-2466

31. Bräunig J, Mergler S, Jyrch S, Hoefig CS, Rosowski M, Mittag $\mathrm{J}$ et al (2018) 3-Iodothyronamine activates a set of membrane 
proteins in murine hypothalamic cell lines. Front Endocrinol (Lausanne) 9:523

32. Mayer CM, Fick LJ, Gingerich S, Belsham DD (2009) Hypothalamic cell lines to investigate neuroendocrine control mechanisms. Front Neuroendocrinol 30(3):405-423

33. Suh SW, Aoyama K, Chen Y, Garnier P, Matsumori Y, Gum E (2003) Hypoglycemic neuronal death and cognitive impairment are prevented by poly(ADP-ribose) polymerase inhibitors administered after hypoglycemia. J Neurosci 23(33):10681-11090

34. Haythorne E, Hamilton DL, Findlay JA, Beall C, McCrimmon RJ, Ashford ML (2016) Chronic exposure to KATP channel openers results in attenuated glucose sensing in hypothalamic GT1-7 neurons. Neuropharmacology 111:212-222

35. Beall C, Hamilton DL, Gallagher J, Logie L, Wright K, Soutar MP et al (2012) Mouse hypothalamic GT1-7 cells demonstrate AMPK-dependent intrinsic glucose-sensing behaviour. Diabetologia 55(9):2432-2444

36. Routh VH, Hao L, Santiago AM, Sheng Z, Zhou C (2014) Hypothalamic glucose sensing: making ends meet. Front Syst Neurosci 8:236. https://doi.org/10.3389/fnsys.2014.00236

37. Tkacs NC, Pan Y, Raghupathi R, Dunn-Meynell AA, Levin BE (2005) Cortical Fluoro-Jade staining and blunted adrenomedullary response to hypoglycemia after noncoma hypoglycemia in rats. J Cereb Blood Flow Metab 25(12):1645-1655

38. Watts AG, Khan AM, Sanchez-Watts G, Salter D, Neuner CM (2006) Activation in neural networks controlling ingestive behaviors: what does it mean, and how do we map and measure it? Physiol Behav 89(4):501-510

39. Evans SB, Wilkinson CW, Gronbeck P, Bennett JL, Taborsky GJJ, Figlewicz DP (2003) Inactivation of the PVN during hypoglycemia partially simulates hypoglycemia-associated autonomic failure. Am J Physiol Regul Integr Comp Physiol 284(1):R57-R65

40. Evans SB, Wilkinson CW, Gronbeck P, Bennett JL, Zavosh A, Taborsky GJJ (2004) Inactivation of the DMH selectively inhibits the ACTH and corticosterone responses to hypoglycemia. Am J Physiol Regul Integr Comp Physiol 286(1):R123-R128

41. Routh VH (2010) Glucose sensing neurons in the ventromedial hypothalamus. Sensors (Basel) 10(10):9002-9025

42. Oomura Y, Kimura K, Ooyama H, Maeno T, Iki M, Kuniyoshi M (1964) Reciprocal activities of the ventromedial and lateral hypothalamic areas of cats. Science 143(3605):484-485
43. Oomura Y, Ooyama H, Sugimori M, Nakamura T, Yamada Y (1974) Glucose inhibition of the glucose-sensitive neurons in the rat lateral hypothalamus. Nature 247(5439):284-286

44. Muroya S, Yada T, Shioda S, Takigawa M (1999) Glucose-sensitive neurons in the rat arcuate nucleus contain neuropeptide $\mathrm{Y}$. Neurosci Lett 264(1-3):113-116

45. Kurita H, Xu KY, Maejima Y, Nakata M, Dezaki K, Santoso P et al (2015) Arcuate $\mathrm{Na}+, \mathrm{K}+$-ATPase senses systemic energy states and regulates feeding behavior through glucose-inhibited neurons. Am J Physiol Endocrinol Metab 309(4):E320-E333

46. Qi L, Kang K, Zhang C, van Dam RM, Kraft P, Hunter D et al (2011) Fat mass-and obesity-associated (FTO) gene variant is associated with obesity: longitudinal analyses in two cohort studies and functional test. Diabetes 57(11):3145-3151

47. Fischer J, Koch L, Emmerling C, Vierkotten J, Peters T, Brüning JC et al (2009) Nature. 458(7240):894-8. doi: 10.1038/ nature 07848. Epub 2009 Feb 22. Inactivation of the Fto gene protects from obesity. Nature 458(7240):894-8.

48. Church C, Moir L, McMurray F, Girard C, Banks GT, Teboul L et al (2010) Overexpression of Fto leads to increased food intake and results in obesity. Nat Genet 42(12):1086-1092

49. Han Z, Niu T, Chang J, Lei X, Zhao M, Wang Q et al (2010) Crystal structure of the FTO protein reveals basis for its substrate specificity. Nature 464(7292):1205-1209

50. Almén MS, Jacobsson JA, Moschonis G, Benedict C, Chrousos GP, Fredriksson R et al (2012) Genome wide analysis reveals association of a FTO gene variant with epigenetic changes. Genomics 99(3):132-137

51. Ma Y (2018) The challenge of microRNA as a biomarker of epilepsy. Curr Neuropharmacol 16(1):37-42

52. Tavakolizadeh J, Roshanaei K, Salmaninejad A, Yari R, Nahand JS, Sarkarizi HK et al (2018) MicroRNAs and exosomes in depression: potential diagnostic biomarkers. J Cell Biochem 119(5):3783-3797

53. Zendjabil M, Favard S, Tse C, Abbou O, Hainque B (2017) The microRNAs as biomarkers: what prospects. C R Biol 340(2):114-131

Publisher's Note Springer Nature remains neutral with regard to jurisdictional claims in published maps and institutional affiliations. 